\title{
Collaboration transnationale en médecine d'urgence
}

\author{
Ulrich Bürgi ${ }^{a}$, \\ Michael Christ ${ }^{b}$, \\ Barbara Hoganc, \\ Wilhelm Behringer ${ }^{\text {, }}$ \\ Christoph Dodt ${ }^{e}$
}

a Président de la Société suisse de médecine d'urgence et de sauvetage (SSMUS), Aarau

b $2^{\mathrm{e}}$ président de la Deutsche Gesellschaft für Interdisziplinäre Notfall- und

Akutmedizin (DGINA), Nuremberg

c Présidente de l'European Society of Emergency Medicine (EuSEM), Hambourg

d Président de l'Austrian Association of Emergency Medicine (AAEM), Vienne

e Président de la Deutsche Gesellschaft für Interdisziplinäre Notfall- und Akutmedizin (DGINA), Munich
Correspondance: Dr Ulrich Bürgi Chef du service d'urgence interdisciplinaire Hôpital cantonal CH-5001 Aarau Tél. 0628385428 ulrich.buergi[at]ksa.ch

\section{Introduction}

On estime à 1,5 millions le nombre des patients qui ont été soignés dans les services d'urgence suisses en 2012. Depuis près de 20 ans, le nombre des admissions aux urgences n'a cessé d'augmenter en Suisse et cette hausse se poursuivra ces prochaines années. Les causes en sont multifactorielles et diffèrent selon les régions et le mandat hospitalier: changements démographiques (croissance, pyramide des âges, groupes ethniques minoritaires), recul de la médecine de premier recours (en particulier dans les zones urbaines), conscience plus élevée de la population en matière de santé et accès facilité aux prestations de santé dans les permanences et les cabinets d'urgence en dehors des heures d'exploitation normales. Les services d'urgence suisses sont surchargés par l'augmentation constante des admissions de patients et fournissent les prestations nécessaires 24 heures sur 24 au prix d'efforts intenses du personnel.

La Société suisse de médecine d'urgence et de sauvetage (SSMUS) travaille depuis plus de 20 ans à l'obtention d'améliorations techniques et structurelles dans les services d'urgence suisses. Dans notre pays, les problèmes sont les mêmes qu'en Allemagne et en Autriche. C'est pourquoi, la SSMUS a décidé de collaborer plus étroitement avec la Deutsche Gesellschaft für Interdisziplinäre Notfall- und Akutmedizin (DGINA) et l'Austrian Association of Emergency Medicine (AAEM) afin d'élaborer des mesures techniques et structurelles dans des concepts et programmes communs.

\section{Cinq thèses pour le développement de la médecine d'urgence en Allemagne, en Autriche et en Suisse}

\section{Situation initiale}

La prise en charge urgente 24 heures sur 24 de patients présentant des troubles de santé aigus est un service médical indispensable pour la population. Au cours des dernières années, le nombre de patients qui ont eu recours à des soins de médecine d'urgence dans un hôpital a énormément augmenté. A l'heure actuelle, entre l'Allemagne, l'Autriche et la Suisse, ce sont près de 25 millions de personnes ( $20 \%$ de la population) qui reçoivent des soins de médecine d'urgence dans les hôpitaux. Les patients concernés ont droit à des soins rapides, adaptés aux risques et de grande qualité pour les troubles de santé ressentis comme une menace. Les conditions-cadres en ma-

\section{Résumé}

D'année en année, le nombre croissant de patients dans les services d'urgence suisses représente une charge de plus en plus grande pour leurs collaborateurs et l'infrastructure des hôpitaux. La Société suisse de médecine d'urgence et de sauvetage (SSMUS) travaille depuis plus de $\mathbf{2 0}$ ans à l'obtention d'améliorations techniques et structurelles en médecine d'urgence et de sauvetage. Comme les problèmes sont similaires dans les pays voisins, notamment en Allemagne et en Autriche, les sociétés de médecine d'urgence des 3 pays (DGINA, AAEM et SSMUS) ont décidé de collaborer plus étroitement. Elles présentent, dans cet article, 5 thèses sur le développement de la médecine d'urgence. L'occasion est également saisie pour préciser les définitions de «cas d'urgence» et de «médecine d'urgence».

tière de politique sanitaire et d'économie exigent en plus une prise charge médicale d'urgence efficiente et efficace.

Dans notre pays, les problèmes sont les mêmes qu'en Allemagne et en Autriche.

Le changement sociodémographique mène chez de nombreux patients en détresse à une augmentation de la multimorbidité et de la complexité médicale, ce qui représente un défi interdisciplinaire particulier qui ne peut être relevé par la médecine hautement spécialisée et sa segmentation croissante ainsi que sa répartition des tâches. Le défi d'une pratique liée aux symptômes et holistique dans le diagnostic et la thérapie exige des structures et des formes d'organisation spéciales ainsi qu'une formation spécifique en médecine d'urgence. C'est pourquoi, dans la plupart des pays du monde, la médecine d'urgence est depuis 


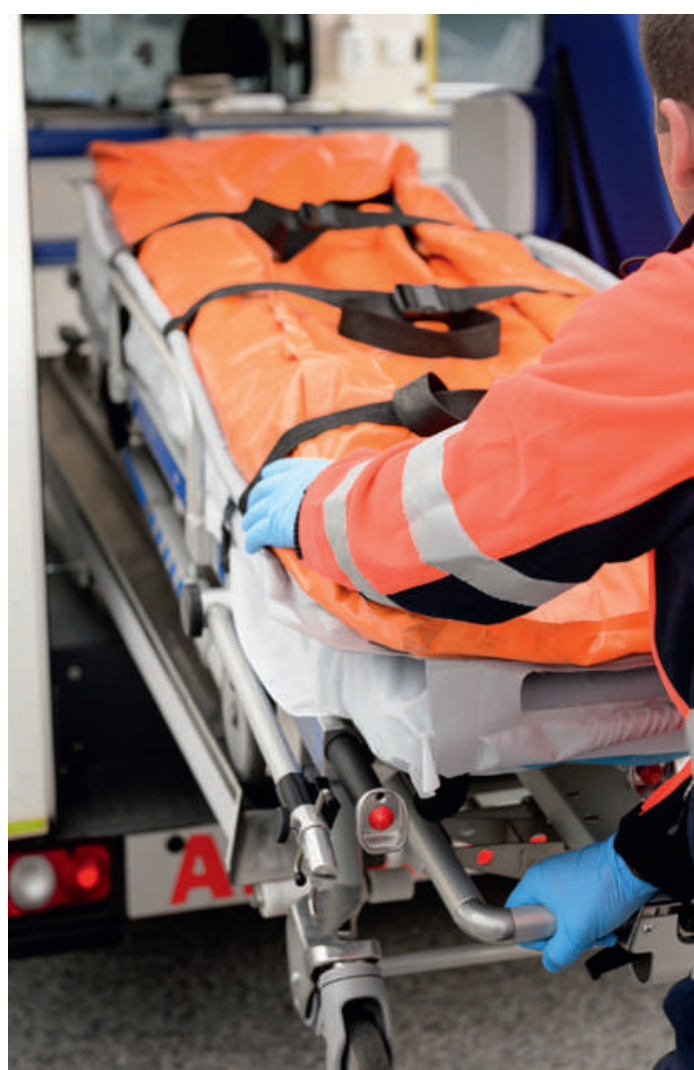

La prise en charge urgente 24 heures sur 24 de patients présentant des troubles de santé aigus est un service médical indispensable pour la population.

des années une spécialité à part entière. La médecine d'urgence est une réalité de fait.

Les sociétés de médecine d'urgence d'Allemagne, d'Autriche et de Suisse (DGINA, AAEM, SSMUS) publient les 5 thèses suivantes:

1. Le développement de la médecine d'urgence au niveau clinique et structurel ainsi que dans la formation postgraduée et continue, en tant que spécialité à part entière avec ses propres structures et formes d'organisation, est une condition préalable à une excellente prise en charge médicale d'urgence.

2. Introduction du titre de spécialiste en médecine d'urgence avec une formation postgraduée de cinq ans.

3. Recommandation des sociétés que les infirmiers spécialisés en médecine d'urgence fassent au plus tôt partie intégrante de l'équipe soignante dans toutes les admissions ou services d'urgence, en Allemagne, en Autriche et en Suisse.

4. L'AAEM, la DGINA et la SSMUS visent à introduire ces prochaines années la saisie et l'analyse des paramètres de qualité de la médecine d'urgence.

5. Etablissement et encouragement de la recherche universitaire et de l'enseignement pour la branche médecine d'urgence.

\section{Comité de la SSMUS}

Dr Mathias Zürcher, p.-d., vice-président, Bâle; Prof. François Sarasin, vice-président, Genève; Prof. Roland Bingisser, Bâle; Dr Ünal Can, Zurich; Dr Adam-Scott Feiner, Lausanne; Dr Walter Hanhart, Neuchâtel; Gabriela Kaufmann, Berne; Prof. Dagmar Keller, Zurich; Dr Beat Lehmann, Berne; Dr Stefan Müller, Zurich; Dr Peter Rupp, Spiez; Dr Barbara Schild, Bellinzona; Dr Robert Sieber, St-Gall; Dr Simon Sulser, Winterthour.

En outre, la mise en application de ces thèses garantira, en Allemagne, en Autriche et en Suisse, une excellente prise en charge médicale d'urgence selon des standards internationaux, malgré les restrictions économiques. Du même coup, il sera également possible d'appliquer la déclaration de politique générale de «l'Union Européenne des Médecins Spécialistes» (UEMS), dans la version du 20 avril 2013, dans les pays germanophones (voir sous www.dgina.de/ media/nachricht/2013_policy_statement_UEMS. pdf).

\section{Définition}

\section{Cas d'urgence médicale}

Par cas d'urgence médicale ou patient en détresse, on entend toute personne présentant des modifications physiques ou mentales dans son état de santé pour lesquelles le patient ou une personne tierce considère comme indispensable une prise en charge médicale et sanitaire immédiate.

\section{La spécialité de la médecine d'urgence}

La médecine d'urgence est une spécialité qui regroupe les connaissances et les aptitudes pratiques permettant de prévenir ou de diagnostiquer et traiter des maladies aiguës et des blessures nécessitant des soins urgents. La spécialité traite d'un large éventail de troubles physiques et comportementaux de patients en détresse. La médecine d'urgence n'est pas définie par le lieu de l'intervention médicale d'urgence mais par le contenu spécialisé «médecine d'urgence» et comprend donc les situations d'urgence préhospitalières et hospitalières.

En médecine d'urgence, la rapidité de la prise en charge est d'une importance cruciale. La pratique hospitalière de la médecine d'urgence comprend le triage préhospitalier et hospitalier, la première évaluation, la stabilisation aiguë des patients concernés et la gestion des cas urgents et critiques jusqu'à leur départ vers les soins ambulatoires ou au transfert vers un autre médecin, une institution ambulatoire ou stationnaire ou d'autres collaborateurs du système de santé. La spécialité comprend aussi la participation au développement des systèmes d'urgence préhospitaliers et la gestion des afflux massifs de blessés ou de malades. 